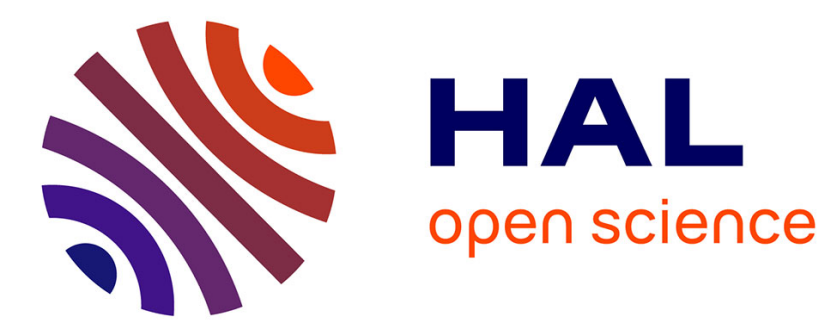

\title{
Global asymptotic stability for an age-structured model of hematopoietic stem cell dynamics
}

\author{
Mostafa Adimy, Abdennasser Chekroun, Tarik-Mohamed Touaoula
}

\section{To cite this version:}

Mostafa Adimy, Abdennasser Chekroun, Tarik-Mohamed Touaoula. Global asymptotic stability for an age-structured model of hematopoietic stem cell dynamics. Applicable Analysis, 2016, pp.1 - 12. 10.1080/00036811.2016.1139698 . hal-01396691

\section{HAL Id: hal-01396691 \\ https://hal.inria.fr/hal-01396691}

Submitted on 15 Nov 2016

HAL is a multi-disciplinary open access archive for the deposit and dissemination of scientific research documents, whether they are published or not. The documents may come from teaching and research institutions in France or abroad, or from public or private research centers.
L'archive ouverte pluridisciplinaire HAL, est destinée au dépôt et à la diffusion de documents scientifiques de niveau recherche, publiés ou non, émanant des établissements d'enseignement et de recherche français ou étrangers, des laboratoires publics ou privés. 


\title{
Global asymptotic stability for an age-structured model of hematopoietic stem cell dynamics
}

\author{
Mostafa Adimy ${ }^{\mathrm{a}}$, Abdennasser Chekroun $^{\mathrm{a}}$, Tarik-Mohamed Touaoula ${ }^{\mathrm{b}}$ \\ ${ }^{a}$ Inria, Université de Lyon, Université Lyon 1, Institut Camille Jordan, 43 Bd. du 11 novembre 1918, \\ F-69200 Villeurbanne Cedex, France \\ ${ }^{b}$ Department of Mathematics, University Aboubekr Belkaid, Tlemcen, Algeria
}

\begin{abstract}
We investigate a system of two nonlinear age-structured partial differential equations describing the dynamics of proliferating and quiescent hematopoietic stem cell populations. The method of characteristics reduces the age-structured model to a system of coupled delay differential and renewal difference equations with continuous time and distributed delay. By constructing a Lyapunov-Krasovskii functional, we give a necessary and sufficient condition for the global asymptotic stability of the trivial steady state, which describes the population dying out. We also give sufficient conditions for the existence of unbounded solutions, which describes the uncontrolled proliferation of hematopoietic stem cell population. This study may be helpful in understanding the behavior of hematopoietic cells in some hematological disorders.
\end{abstract}

Keywords: Age-structured PDE; delay differential-difference system; Lyapunov-Krasovskii functional; cell dynamics; hematopoietic stem cells

AMS Subject Classifications: 34D20; 34D23; 34K06; 92C37

\section{Introduction}

Many biological systems are self-regulating and hence, they involve nonlocal terms (called delays) in feedback loops. These delays are due to the inherent time lag between the sensing and control signals. The retardation usually originates from maturing processes or finite signaling velocities. Sometimes the delays can be of considerable length and cannot be neglected. In general, the delays can represent incubation periods [11], gestation times [9], cell division processes [19], or can simply be used to model unknown intermediate steps in reaction chains, accounting only for the time required for these processes to occur.

A classical approach to introduce delays in differential systems starts from certain agestructured models (transport PDE). The age-structured systems can be transformed in a natural way using the method of characteristics to a system of threshold-type delay differential-difference equations (see $[6,18,24])$. This approach is used in this paper to reduce an age-structured model to a system composed with a delay differential equation and a renewal difference equation with continuous time and distributed delay.

Mathematical modeling of hematopoietic stem cell (HSC) dynamics has been extensively studied in the past 40 years. In 1978, Mackey [19] proposed a mathematical model of HSC

Email addresses: mostafa.adimy@inria.fr (Mostafa Adimy), chekroun@math.univ-lyon1.fr (Abdennasser Chekroun), tarik.touaoula@mail.univ-tlemcen.dz (Tarik-Mohamed Touaoula)

Corresponding author: Mostafa Adimy 
dynamics formed by a system of two delay differential equations. This system describes the evolution of proliferating and quiescent HSCs. The delay describes the average cell cycle duration. The model of Mackey stressed the influence of some factors, such as the apoptotic rate, the introduction rate from the quiescent phase into the proliferating phase, and the cell cycle duration, playing an important role in the behavior of the system. Since then, Mackey's model has been improved by many authors. It has been analyzed by Pujo-Menjouet and Mackey [22], and Pujo-Menjouet et al. [21], in order to prove the existence of long period oscillations, characterizing situations observed in chronic myelogenous leukemia, a cancer of HSCs. Many authors, including $[2,3,4,5,6,7,8]$ and the references therein, analyzed various versions of Mackey's model and investigated the effect of perturbations of the parameters of the system on the behavior of the cell population. In all these studies, the authors assumed that just after division all cells enter immediately the quiescent phase. This restrictive assumption allows to reduce the model to a delay differential system.

In the general situation in a cellular population and in particular, in HSC population (see $[10,25])$, it is believed that just after cell division the daughter cells follow one of the two pathways: they enter the quiescent phase or they return to the cell cycle to divide again. Contrary to the above works, we take into account this situation. We reconsider the model of Mackey and we suppose that just after division only a part of daughter cells enter the quiescent phase (longterm proliferation) and the other part of cells return immediately to the proliferating phase to divide again (short-term proliferation). This assumption leads to a modification of Mackey's model, which cannot be reduced to a classical delay differential system as in the previous works. We obtain a system of coupled delay differential and continuous time difference equations with distributed delay. We focus our mathematical analysis on this delay differential-difference system. We show that the behavior of the system is related to both short and long proliferating terms. We focus on the influence of coefficients of the system on the global asymptotic stability of the trivial steady state and the existence of unbounded solutions. This model may help to explain the behavior of HSCs in some hematological diseases.

In the next section (Section 2), we present the age-structured model that will be considered in this paper. In Section 3, we use the characteristics method to reduce the model to a coupled delay differential and renewal difference equations with continuous time and distributed delay. In Section 4, we give a necessary and sufficient condition for the global asymptotic stability of the trivial steady state. In Section 5, we establish the existence of unbounded solutions of the system.

\section{Age-structured model of HSC dynamics}

Let consider a population of HSCs, located in the bone marrow. The period of life of each HSC is divided into two phases: quiescence and proliferation (see [19]). Denote by $q(t, a)$ (respectively, $p(t, a)$ ) the density of quiescent (respectively, proliferating) HSCs with time $t \geq 0$ and age $a$. The age represents the time spent by a cell in one of the two phases, thus it ranges, in quiescent phase, from 0 to infinity (which means that, cells can stay their entire life in the quiescent phase), and in the proliferating phase from 0 to $\tau>0$. Quiescent cells cannot divide, and provide they not died (with a rate $\delta \geq 0$ witch take into account the differentiation), they can be introduced in the proliferating phase with a rate $\beta \geq 0$. The nature of the trigger signal for introduction in the proliferating phase is not clear. However, the work of Sachs [23] (see also [19]) shows that we can reasonably think that it strongly depends on the entire quiescent cell population, that is, $\beta:=\beta(Q(t))$ with

$$
Q(t)=\int_{0}^{+\infty} q(t, a) \mathrm{d} a, \quad t \geq 0 .
$$


The function $\beta$ is assumed to be differentiable and strictly decreasing with $\lim _{Q \rightarrow+\infty} \beta(Q)=0$. Typically, $\beta$ is a Hill function (see $[19,21,22]$ ). The proliferating phase is the active part of the cellular development. As soon as cells enter the proliferating phase, they perform a series of process leading to division at mitosis before reaching the maximum age $a=\tau$. If they do not die by apoptosis (programmed cell death) with a rate $\gamma \geq 0$, they are committed to divide with a rate $d_{\tau}(a)$ depending on their age $0<a<\tau$. The function $d_{\tau}$ is assumed to be $L_{\text {loc }}^{1}\left([0, \tau), \mathbb{R}^{+}\right)$ such that

$$
\int_{0}^{\tau} d_{\tau}(a) \mathrm{d} a=+\infty
$$

This later assumption describes the fact that cells which did not die have to divide before they reach the maximal age $\tau$. We suppose that just after division, a fraction $(1-K)$ with $K \in[0,1]$, of daughter cells enter the quiescent phase (long-term proliferation) and the other fraction $K$ of daughter cells return immediately to the proliferating phase to divide again (shortterm proliferation). Then, the part of dividing cells with age $a \in(0, \tau)$ that enter the quiescent phase is $2(1-K) d_{\tau}(a)$ and the part that return to the proliferating phase is $2 K d_{\tau}(a)$.

Then the densities $q(t, a)$ and $p(t, a)$ satisfy, for $t>0$, the system of age-structured partial differential equations

$$
\begin{cases}\frac{\partial q(t, a)}{\partial t}+\frac{\partial q(t, a)}{\partial a}=-(\delta+\beta(Q(t))) q(t, a), & a>0 \\ \frac{\partial p(t, a)}{\partial t}+\frac{\partial p(t, a)}{\partial a}=-\left(\gamma+d_{\tau}(a)\right) p(t, a), & 0<a<\tau \\ q(t, 0)=2(1-K) \int_{0}^{\tau} d_{\tau}(a) p(t, a) \mathrm{d} a, & \\ p(t, 0)=\int_{0}^{+\infty} \beta(Q(t)) q(t, a) \mathrm{d} a+2 K \int_{0}^{\tau} d_{\tau}(a) p(t, a) \mathrm{d} a, & \\ \lim _{a \rightarrow+\infty} q(t, a)=0 .\end{cases}
$$

The initial conditions are nonnegative functions given by

$$
\left\{\begin{array}{l}
q(0, a)=q_{0}(a), \quad a>0, \\
p(0, a)=p_{0}(a), \quad 0<a<\tau .
\end{array}\right.
$$

We assume that

$$
q_{0} \in W^{1,1}(0,+\infty), \quad p_{0} \in W^{1,1}(0, \tau) \quad \text { and } \quad d_{\tau}(\cdot) p_{0}(\cdot) \in L^{1}(0, \tau),
$$

where $W^{1,1}(a, b)$ is the space of $L^{1}$-functions on the interval $(a, b)$ such that there derivatives are also $L^{1}$-functions on $(a, b)$.

The two boundary conditions in (2), for $a=0$, describe the flux of cells entering each phase: new proliferating cells are quiescent cells introduced with a rate $\beta$ and cells coming from the division of proliferating cells that return to the cell cycle, with a rate $K d_{\tau}$, to divide again, and new resting cells are coming from the other part of dividing cells (with a rate $(1-K) d_{\tau}$ ). It is important to note that the boundary conditions in (2), for $a=0$ and $a \rightarrow+\infty$, are not required to hold at $t=0$ (see $[17,14]$ ). Indeed, these relations are satisfied at $t=0$ if and only if the 
initial functions $q_{0}$ and $p_{0}$ satisfy the following compatibility condition

$$
\left\{\begin{array}{l}
q_{0}(0)=2(1-K) \int_{0}^{\tau} d_{\tau}(a) p_{0}(a) \mathrm{d} a \\
p_{0}(0)=\beta\left(Q_{0}\right) Q_{0}+2 K \int_{0}^{\tau} d_{\tau}(a) p_{0}(a) \mathrm{d} a \\
\lim _{a \rightarrow+\infty} q_{0}(a)=0,
\end{array}\right.
$$

where

$$
Q_{0}=\int_{0}^{+\infty} q_{0}(a) \mathrm{d} a .
$$

The assumptions (4) and (5) provide us the existence of a solution of (2) (see [17]); actually, the assumption (4) is enough to provide a solution of (2) in the sense stated in Theorem 4.3 on page 16 of [17].

The treatment of the system (2) will be developed in the next sections; in fact, instead of studying (2) directly, it will be transformed into a delay differential-difference system which is derived in the next section.

\section{Reduction to a coupled retarded functional differential equations}

By using the method of characteristics (see $[17,26]$ ), the solutions $p(t, a)$ of $(2)$ are given, for $0<a<\tau$, by

$$
p(t, a)= \begin{cases}e^{-\gamma t} \frac{\Pi_{\tau}(a)}{\Pi_{\tau}(a-t)} p(0, a-t), & 0<t \leq a, \\ e^{-\gamma a} \Pi_{\tau}(a) p(t-a, 0), & t>a,\end{cases}
$$

with

$$
\Pi_{\tau}(a):=\exp \left(-\int_{0}^{a} d_{\tau}(s) \mathrm{d} s\right), 0<a<\tau .
$$

Set

$$
D_{\tau}(a):=d_{\tau}(a) \Pi_{\tau}(a), \quad 0<a<\tau .
$$

One can check that $a \mapsto D_{\tau}(a)$ represents the density of division of proliferating cells, i.e. $D_{\tau}(a)$ is the probability density for a cell to divide at age $a$. In particular, thanks to (12) we have

$$
\int_{0}^{\tau} D_{\tau}(a) \mathrm{d} a=1 .
$$

Integrating system (2) over the age, remembering that $a>0$ in the quiescent phase and $0<$ $a<\tau$ is the proliferating phase, and by using the boundary and initial conditions, we obtain the following system 


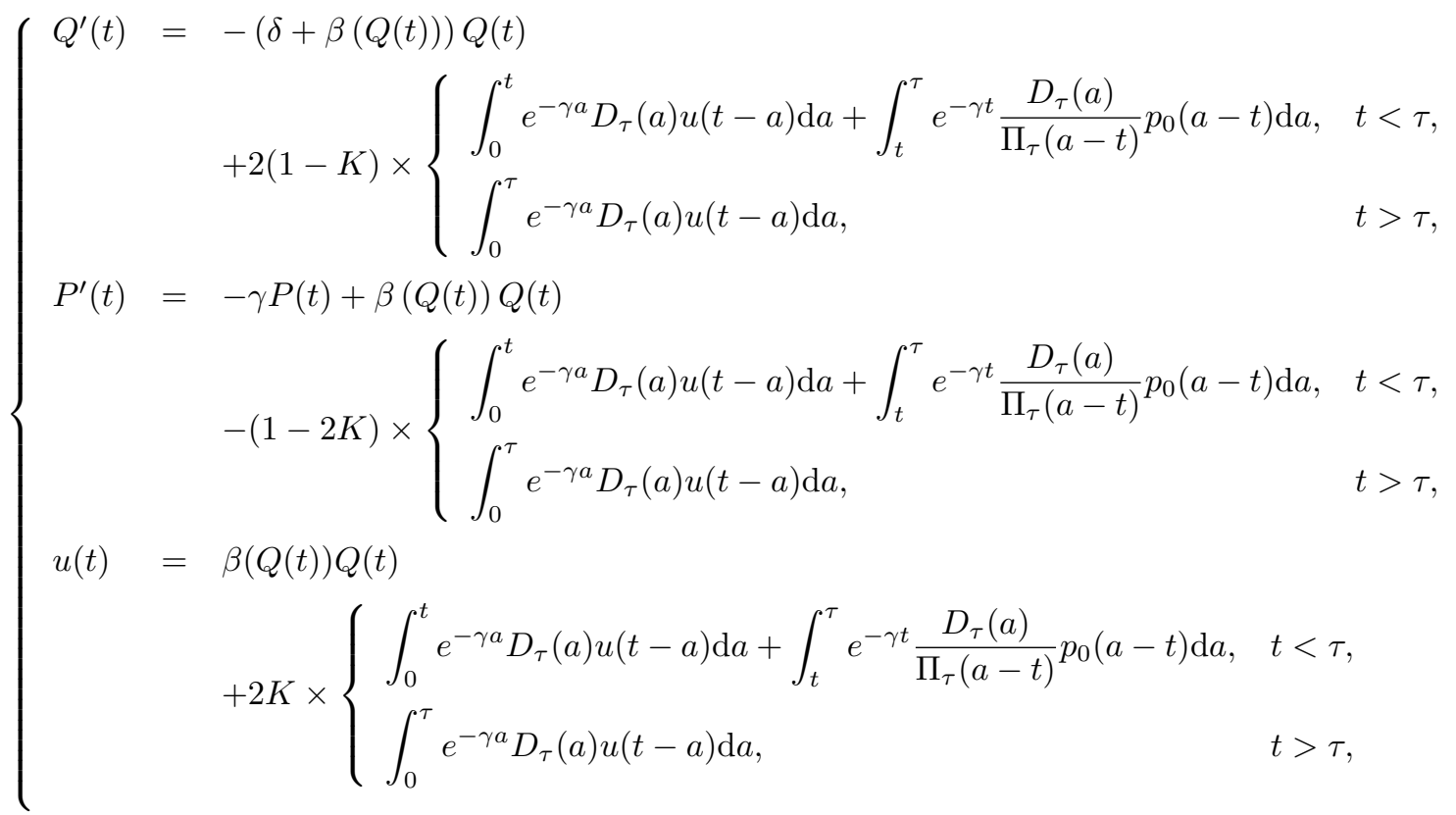

where $u(t)=p(t, 0)$ is the new proliferating cells and $P(t)=\int_{0}^{\tau} p(t, a) \mathrm{d} a$ is the total population of the proliferating cells. The initial conditions become

$$
Q(0)=Q_{0}:=\int_{0}^{+\infty} q_{0}(a) \mathrm{d} a \text { and } P(0)=P_{0}:=\int_{0}^{\tau} p_{0}(a) \mathrm{d} a .
$$

We put, for $t \in[-\tau, 0]$,

$$
\phi(t):=\frac{e^{-\gamma t}}{\Pi_{\tau}(-t)} p_{0}(-t) .
$$

Then, system (7) can be written, for $t>0$,

$$
\left\{\begin{array}{l}
Q^{\prime}(t)=-(\delta+\beta(Q(t))) Q(t)+2(1-K) \int_{0}^{\tau} e^{-\gamma a} D_{\tau}(a) u(t-a) \mathrm{d} a, \\
P^{\prime}(t)=-\gamma P(t)+\beta(Q(t)) Q(t)-(1-2 K) \int_{0}^{\tau} e^{-\gamma a} D_{\tau}(a) u(t-a) \mathrm{d} a \\
u(t)=\beta(Q(t)) Q(t)+2 K \int_{0}^{\tau} e^{-\gamma a} D_{\tau}(a) u(t-a) \mathrm{d} a
\end{array}\right.
$$

with initial conditions

$$
Q(0)=Q_{0}, \quad P(0)=P_{0} \quad \text { and } \quad u(t)=\phi(t) \text { for } \quad-\tau \leq t \leq 0 .
$$

Thanks to (6) and (8), we obtain

$$
P(t)=\int_{0}^{\tau} e^{-\gamma a} \Pi_{\tau}(a) u(t-a) \mathrm{d} a, \quad t>0 .
$$


Since $Q$ and $u$ do not depend on $P$, we will focus on the study of the system

$$
\left\{\begin{array}{l}
Q^{\prime}(t)=-(\delta+\beta(Q(t))) Q(t)+2(1-K) \int_{0}^{\tau} e^{-\gamma a} D_{\tau}(a) u(t-a) \mathrm{d} a, \\
u(t)=\beta(Q(t)) Q(t)+2 K \int_{0}^{\tau} e^{-\gamma a} D_{\tau}(a) u(t-a) \mathrm{d} a
\end{array}\right.
$$

with initial conditions given by

$$
Q(0)=Q_{0} \text { and } u(t)=\phi(t) \text { for }-\tau \leq t \leq 0 .
$$

System (10) is a coupled of differential and difference equations with distributed delay.

The case when $d_{\tau}$ is a Dirac distribution concentrated at $\tau$,

$$
d_{\tau}(a)=\delta_{\tau}(a):= \begin{cases}+\infty, & a=\tau, \\ 0, & a \neq \tau,\end{cases}
$$

was considered in the previous work [1], in the special case where $\gamma=0$. In that case, we have

$$
\int_{0}^{\tau} d_{\tau}(a) \mathrm{d} a=1
$$

and the integral in the system (10) takes the form

$$
\int_{0}^{\tau} e^{-\gamma a} D_{\tau}(a) u(t-a) \mathrm{d} a=e^{-\gamma \tau} u(t-\tau) .
$$

Then, the system (10) can be reduced to a delay differential-difference model with a discrete delay (see [1]).

In all this paper, we assume that $Q_{0} \geq 0, \phi$ is a nonnegative and continuous function on $[-\tau, 0], Q \mapsto \beta(Q) Q$ is a Lipschitz-continuous function, and the following inequality

$$
2 K \int_{0}^{\tau} e^{-\gamma a} D_{\tau}(a) \mathrm{d} a<1 .
$$

Then, the existence and uniqueness of nonnegative solutions of (10)-(11) can be obtained in the same manner as in [15].

From a solution $(Q, u)$ of (10)-(11), we can obtain a solution of (2). Indeed, under the conditions (4) and (5), we can proceed as in Theorem 4.2 on page 15 and Theorem 2.3 on page 51 of [17], to prove that the couple of functions $(q, p)$ defined by

$$
q(t, a)= \begin{cases}q_{0}(a-t) \exp \left(-\int_{a-t}^{a}(\delta+\beta(Q(s))) \mathrm{d} s\right), & 0<t \leq a, \\ 2(1-K)\left(\int_{0}^{\tau} e^{-\gamma s} D_{\tau}(s) u(t-s) \mathrm{d} s\right) \exp \left(-\int_{0}^{a}(\delta+\beta(Q(s))) \mathrm{d} s\right), & t>a,\end{cases}
$$

and

$$
p(t, a)= \begin{cases}e^{-\gamma t} \frac{\Pi_{\tau}(a)}{\Pi_{\tau}(a-t)} p_{0}(a-t), & 0<t \leq a, \\ e^{-\gamma a} \Pi_{\tau}(a) u(t-a), & t>a,\end{cases}
$$

satisfies the system (2). 


\section{Global asymptotic stability of the trivial steady state}

Consider the auxiliary linear homogeneous difference equation

$$
u(t)=\mathcal{D}\left(u_{t}\right), \quad t \geq 0,
$$

where the history function $u_{t} \in C([-\tau, 0], \mathbb{R})$ is defined, for $t \geq 0$ and $u \in C([-\tau,+\infty), \mathbb{R})$, by $u_{t}(\theta)=u(t+\theta)$ for $\theta \in[-\tau, 0]$, and the operator $\mathcal{D}: C([-\tau, 0], \mathbb{R}) \rightarrow \mathbb{R}$ is given by

$$
\mathcal{D}(\psi)=2 K \int_{0}^{\tau} e^{-\gamma a} D_{\tau}(a) \psi(-a) \mathrm{d} a .
$$

Remark that the condition (13) is equivalent to

$$
\|\mathcal{D}\|:=\sup _{\|\psi\| \leq 1}|\mathcal{D}(\psi)|<1,
$$

with $\|\psi\|=\sup _{\theta \in[-\tau, 0]}|\psi(\theta)|$. The condition (15) says that the zero solution of the linear difference equation (14) is globally asymptotically stable (see Theorem 3.5 on page 275 of [16]).

The second equation of (10) can be written

$$
u(t)-\mathcal{D}\left(u_{t}\right)=\beta(Q(t)) Q(t), \quad t>0 .
$$

We put

$$
\nu_{\gamma}(\tau)=\int_{0}^{\tau} e^{-\gamma a} D_{\tau}(a) \mathrm{d} a .
$$

Then, the inequality (13) (or (15)) becomes

$$
2 K \nu_{\gamma}(\tau)<1 .
$$

As mentioned before, we investigate the global asymptotic stability of the trivial steady state using a Lyapunov-Krasovskii functional approach and a notion called input-to-state stability property of system (10) (see $[13,20]$ ).

The following lemma gives a fundamental inequality that leads to the input-to-state stability property of system (10).

Lemma 1. Let $(Q, u)$ be the solution of system (10) associated to the initial condition $\left(Q_{0}, \phi\right) \in$ $\mathbb{R} \times C([-\tau, 0], \mathbb{R})$. Then there exist $\alpha, C>0$ such that, for $t>0$,

$$
\begin{aligned}
|u(t)| & \leq C\left[\|\phi\| e^{-\alpha t}+\sup _{0 \leq s \leq t} \mid \beta(Q(s)) Q(s) \|\right], \\
& \leq C\left[\|\phi\| e^{-\alpha t}+\beta_{0} \sup _{0 \leq s \leq t}|Q(s)|\right]
\end{aligned}
$$

with $\|\phi\|=\sup _{\theta \in[-\tau, 0]}|\phi(\theta)|$ and $\beta_{0}:=\beta(0)=\max _{x \geq 0} \beta(x)$.

Proof. This result follows from Theorem 3.5 on page 275 of [16].

We state the following lemma.

Lemma 2. Let $(Q(t), u(t))$ be a solution of (10). Then, $\lim _{t \rightarrow+\infty} u(t)=0$ if and only if $\lim _{t \rightarrow+\infty} \beta(Q(t)) Q(t)=0$. 
Proof. The proof of this result is based on the equation (16), the inequalities (19) and ([12], Lemma 3.5).

Remark that when $\beta: \mathbb{R}^{+} \mapsto \mathbb{R}^{+}$is a Hill function (see $[19,21,22]$ ) given by

$$
\beta(x)=\frac{\beta_{0} \theta^{n}}{x^{n}+\theta^{n}}, \text { with } \beta_{0}>0, \theta>0 \text { and } n>1,
$$

the only possible way to have $\lim _{t \rightarrow+\infty}(\beta(Q(t)) Q(t))=0$ is $\lim _{t \rightarrow+\infty} Q(t)=0$ or $\lim _{t \rightarrow+\infty} Q(t)=$ $+\infty$.

We now prove the result dealing with the global asymptotic stability of the trivial steady state of (10) and its instability.

Theorem 1. (i) Assume that $\delta>0$ and

$$
\frac{\delta}{\beta_{0}}>\frac{2 \nu_{\gamma}(\tau)-1}{1-2 K \nu_{\gamma}(\tau)}
$$

Then, the trivial steady state of (10) is globally asymptotically stable.

(ii) Assume that

$$
\frac{\delta}{\beta_{0}}<\frac{2 \nu_{\gamma}(\tau)-1}{1-2 K \nu_{\gamma}(\tau)}
$$

Then, no nontrivial solution of the system (10) tends to the trivial steady state.

Proof. (i) Consider the continuous functional

$$
\begin{aligned}
V: \mathbb{R}^{+} \times C\left([-\tau, 0], \mathbb{R}^{+}\right) & \rightarrow \mathbb{R}^{+}, \\
\left(Q_{0}, \phi\right) & \mapsto V\left(Q_{0}, \phi\right),
\end{aligned}
$$

defined by

$$
V\left(Q_{0}, \phi\right)=Q_{0}+\frac{2(1-K)}{1-2 K \nu_{\gamma}(\tau)} \int_{0}^{\tau} e^{-\gamma a} D_{\tau}(a) \int_{-a}^{0} \phi(\theta) \mathrm{d} \theta \mathrm{d} a .
$$

It is not difficult to check that

$$
Q_{0} \leq V\left(Q_{0}, \phi\right) \leq \vartheta\left(\left\|\left(Q_{0}, \phi\right)\right\|\right),
$$

with $\left\|\left(Q_{0}, \phi\right)\right\|:=\max \left\{\left|Q_{0}\right|,\|\phi\|\right\}$ and

$$
\vartheta(x)=\left(1+\frac{2(1-K) \tau \nu_{\gamma}(\tau)}{1-2 K \nu_{\gamma}(\tau)}\right) x, \quad x \geq 0 .
$$

Let $(Q, u)$ be a solution of (10). We have

$$
V\left(Q(t), u_{t}\right)=Q(t)+\frac{2(1-K)}{1-2 K \nu_{\gamma}(\tau)} \int_{0}^{\tau} e^{-\gamma a} D_{\tau}(a) \int_{t-a}^{t} u(\theta) \mathrm{d} \theta \mathrm{d} a .
$$

The function $t \mapsto V\left(Q(t), u_{t}\right)$ is differentiable on $(0,+\infty)$ and

$$
\frac{d}{d t} V\left(Q(t), u_{t}\right)=Q^{\prime}(t)+\frac{2(1-K)}{1-2 K \nu_{\gamma}(\tau)} \int_{0}^{\tau} e^{-\gamma a} D_{\tau}(a)[u(t)-u(t-a)] \mathrm{d} a .
$$


Then, using (10) one can check that

$$
\begin{aligned}
\frac{d}{d t} V\left(Q(t), u_{t}\right)= & -(\delta+\beta(Q(t))) Q(t)+2(1-K) \int_{0}^{\tau} e^{-\gamma a} D_{\tau}(a) u(t-a) \mathrm{d} a \\
& +\frac{2(1-K)}{1-2 K \nu_{\gamma}(\tau)} \int_{0}^{\tau} e^{-\gamma a} D_{\tau}(a)\left[\beta(Q(t)) Q(t)+\mathcal{D}\left(u_{t}\right)\right] \mathrm{d} a \\
& -\frac{2(1-K)}{1-2 K \nu_{\gamma}(\tau)} \int_{0}^{\tau} e^{-\gamma a} D_{\tau}(a) u(t-a) \mathrm{d} a
\end{aligned}
$$

It follows that

$$
\begin{aligned}
\frac{d}{d t} V\left(Q(t), u_{t}\right)= & -\left(\delta-\frac{2 \nu_{\gamma}(\tau)-1}{1-2 K \nu_{\gamma}(\tau)} \beta(Q(t))\right) Q(t) \\
& +2(1-K) \int_{0}^{\tau} e^{-\gamma a} D_{\tau}(a) u(t-a) \mathrm{d} a \\
& +\frac{4 K(1-K) \nu_{\gamma}(\tau)}{1-2 K \nu_{\gamma}(\tau)} \int_{0}^{\tau} e^{-\gamma a} D_{\tau}(a) u(t-a) \mathrm{d} a \\
& -\frac{2(1-K)}{1-2 K \nu_{\gamma}(\tau)} \int_{0}^{\tau} e^{-\gamma a} D_{\tau}(a) u(t-a) \mathrm{d} a \\
= & -\left(\delta-\frac{2 \nu_{\gamma}(\tau)-1}{1-2 K \nu_{\gamma}(\tau)} \beta(Q(t))\right) Q(t)
\end{aligned}
$$

Consequently,

$$
\frac{d}{d t} V\left(Q(t), u_{t}\right)=-w(Q(t)) Q(t), \quad t>0
$$

with

$$
w(x)=\left(\delta-\frac{2 \nu_{\gamma}(\tau)-1}{1-2 K \nu_{\gamma}(\tau)} \beta(x)\right), \text { for } x \geq 0 .
$$

If $2 \nu_{\gamma}(\tau)>1$, then from (18) and since the function $\beta$ is decreasing, it follows that $w$ is an increasing function on $[0,+\infty)$. Furthermore, the condition $(21)$ implies that $w(0)>0$. If $2 \nu_{\gamma}(\tau) \leq 1$, then (21) is always satisfied and $w(x) \geq \delta>0$, for all $x \geq 0$. We conclude that $V$ is a Lyapunov-Krasovskii functional. This, together with the input-to-state stability estimation (19), implies the global asymptotic stability of the trivial steady state (see [13], Theorem 3).

(ii) We now suppose that the condition (22) is satisfied. Let $(Q, u)$ be a solution of (10). Assume by contradiction that $Q(t) \rightarrow 0$ as $t \rightarrow \infty$. By the continuity of the function $\beta$, we have $\beta(Q(t)) \rightarrow \beta_{0}:=\beta(0)$ as $t \rightarrow \infty$. Consequently, for all $\epsilon>0$ there exists $A(\epsilon)$ such that, for all $t \geq A(\epsilon)$,

$$
\beta_{0}-\epsilon<\beta(Q(t)) \leq \beta_{0} .
$$

We fix $0<\epsilon<\beta_{0}$. By integration the system (10) over $t \in(A(\epsilon),+\infty)$, we get

$$
\left\{\begin{array}{l}
Q(A(\epsilon))=\int_{A(\epsilon)}^{+\infty}[\delta+\beta(Q(t))] Q(t) \mathrm{d} t-2(1-K) \int_{A(\epsilon)}^{+\infty} \int_{0}^{\tau} e^{-\gamma a} D_{\tau}(a) u(t-a) \mathrm{d} a \mathrm{~d} t \\
\int_{A(\epsilon)}^{+\infty} u(t) \mathrm{d} t=\int_{A(\epsilon)}^{+\infty} \beta(Q(t)) Q(t) \mathrm{d} t+2 K \int_{A(\epsilon)}^{+\infty} \int_{0}^{\tau} e^{-\gamma a} D_{\tau}(a) u(t-a) \mathrm{d} a \mathrm{~d} t .
\end{array}\right.
$$


By applying Fubini's theorem we obtain

$$
\begin{aligned}
\int_{A(\epsilon)}^{+\infty} \int_{0}^{\tau} e^{-\gamma a} D_{\tau}(a) u(t-a) \mathrm{d} a \mathrm{~d} t & =\int_{0}^{\tau} e^{-\gamma a} D_{\tau}(a) \int_{A(\epsilon)-a}^{+\infty} u(t) \mathrm{d} t \mathrm{~d} a \\
& \geq \nu_{\gamma}(\tau) \int_{A(\epsilon)}^{+\infty} u(t) \mathrm{d} t
\end{aligned}
$$

From (23), the second equation of (24) and (25), it follows

$$
\int_{A(\epsilon)}^{+\infty} u(t) \mathrm{d} t \geq\left(\beta_{0}-\epsilon\right) \int_{A(\epsilon)}^{+\infty} Q(t) \mathrm{d} t+2 K \nu_{\gamma}(\tau) \int_{A(\epsilon)}^{+\infty} u(t) \mathrm{d} t .
$$

Then, thanks to (18), we obtain

$$
\int_{A(\epsilon)}^{+\infty} u(t) \mathrm{d} t \geq \frac{\beta_{0}-\epsilon}{1-2 K \nu_{\gamma}(\tau)} \int_{A(\epsilon)}^{+\infty} Q(t) \mathrm{d} t
$$

We deduce from the first equation of (24) and (25), that

$$
\begin{aligned}
Q(A(\epsilon)) & \leq\left(\delta+\beta_{0}\right) \int_{A(\epsilon)}^{+\infty} Q(t) \mathrm{d} t-2(1-K) \nu_{\gamma}(\tau) \int_{A(\epsilon)}^{+\infty} u(t) \mathrm{d} t \\
& \leq\left(\delta-\beta_{0} \frac{2 \nu_{\gamma}(\tau)-1}{1-2 K \nu_{\gamma}(\tau)}+\epsilon \frac{2(1-K) \nu_{\gamma}(\tau)}{1-2 K \nu_{\gamma}(\tau)}\right) \int_{A(\epsilon)}^{+\infty} Q(t) \mathrm{d} t
\end{aligned}
$$

As a consequence of $(22)$, one can choose $\epsilon \in\left(0, \beta_{0}\right)$ such that $Q(A(\epsilon))<0$. This leads to a contradiction with the positivity of the solutions. Then, $Q(t)$ does not tend to zero as $t \rightarrow+\infty$.

Remark 1. 1. One can apply the same reasoning as in the proof of Theorem 1-( $i)$, to obtain the global stability of the trivial steady state (but not the global asymptotic stability) under the condition

$$
\frac{\delta}{\beta_{0}}=\frac{2 \nu_{\gamma}(\tau)-1}{1-2 K \nu_{\gamma}(\tau)}
$$

For the definition of the global stability, see for instance [16].

2. If $\delta=0$ and $\nu_{\gamma}(\tau)<1 / 2$, then (21) is satisfied and the trivial steady state is globally asymptotically stable.

3. If $\delta=0$ and $\nu_{\gamma}(\tau)>1 / 2$, then (22) is satisfied and the nontrivial solutions of the system (10) do not converge to zero.

4. Although the solution $Q(t)$ does not tend to zero, $u(t)$ may tend to zero (for instance, when $Q(t)$ tends to infinity and $\beta$ is a Hill function (20)).

\section{Existence of unbounded solutions}

We now study the special case $\delta=0$. We show in the next proposition, that the solutions of (10) may be unbounded on $[0,+\infty)$.

Proposition 1. Assume that $\delta=0$ and

$$
2(1-K) \nu_{\gamma}(\tau)>1
$$

Let $(Q, u)$ be a solution of (10). Suppose that there exists $\bar{x}>0$ such that the function $x \mapsto \beta(x) x$ is decreasing for $x \geq \bar{x}$. Assume that there exists $\bar{t} \geq 0$ such that $Q(\bar{t})>\bar{x}$ and $Q$ is increasing on $[\bar{t}, \bar{t}+\tau]$. Then, $Q$ is increasing on $[\bar{t},+\infty)$ and $\lim _{t \rightarrow+\infty} Q(t)=+\infty$. 
Proof. Let $a \in[0, \tau]$. We have $\bar{x}<Q(\bar{t}) \leq Q(\bar{t}+\tau-a) \leq Q(\bar{t}+\tau)$. Then, it follows that $\beta(Q(\bar{t}+\tau)) Q(\bar{t}+\tau) \leq \beta(Q(\bar{t}+\tau-a)) Q(\bar{t}+\tau-a)$. Consequently,

$$
\begin{aligned}
Q^{\prime}(\bar{t}+\tau)= & -\beta(Q(\bar{t}+\tau)) Q(\bar{t}+\tau)+2(1-K) \int_{0}^{\tau} e^{-\gamma a} D_{\tau}(a) u(\bar{t}+\tau-a) \mathrm{d} a \\
= & -\beta(Q(\bar{t}+\tau)) Q(\bar{t}+\tau) \\
& +2(1-K) \int_{0}^{\tau} e^{-\gamma a} D_{\tau}(a) \beta(Q(\bar{t}+\tau-a)) Q(\bar{t}+\tau-a) \mathrm{d} a \\
& +4 K(1-K) \int_{0}^{\tau} e^{-\gamma a} D_{\tau}(a) \int_{0}^{\tau} e^{-\gamma s} D_{\tau}(s) u(\bar{t}+\tau-a-s) \mathrm{d} s \mathrm{~d} a, \\
\geq & \left(2(1-K) \nu_{\gamma}(\tau)-1\right) \beta(Q(\bar{t}+\tau)) Q(\bar{t}+\tau), \\
> & 0 .
\end{aligned}
$$

Hence, $Q$ is increasing on $[\bar{t}, T]$ for some $T>\bar{t}+\tau$. Suppose by contradiction that there exists $T>\bar{t}+\tau$ such that $Q^{\prime}(T)=0$ and $Q^{\prime}(t)>0$ for all $t \in[\bar{t}, T)$. Then, $\bar{x}<Q(\bar{t}+\tau)<Q(T)$. We repeat the same calculation as above and we get

$$
0=Q^{\prime}(T) \geq\left(2(1-K) \nu_{\gamma}(\tau)-1\right) \beta(Q(T)) Q(T)>0 .
$$

This gives a contradiction. Then, $Q$ is increasing on $[\bar{t},+\infty)$. We now suppose by contradiction that $Q$ is bounded on $[t,+\infty)$. Consequently, the limit $L:=\lim _{t \rightarrow+\infty} Q(t)$ exists. As $Q$ is an increasing function on $[\bar{t},+\infty)$, we have $\lim _{t \rightarrow+\infty} Q^{\prime}(t)=0$. Letting $t$ tend to infinity in both sides of the system (10), we obtain

$$
\beta(L) L\left(\frac{2 \nu_{\gamma}(\tau)-1}{1-2 K \nu_{\gamma}(\tau)}\right)=0 .
$$

Hence $L=0$. This leads to a contradiction. We conclude that $\lim _{t \rightarrow+\infty} Q(t)=+\infty$.

The term $2(1-K) \nu_{\gamma}(\tau)$ in $(27)$ is the portion of dividing cells that enter the quiescent phase and it is assumed to be greater than 1 , with $\delta=0$. So, it is no surprise that $\lim _{t \rightarrow+\infty} Q(t)=+\infty$.

In the next proposition, we construct an example of functions (3) such that the initial condition (11) of system (10) satisfies the assumptions of Proposition 1. First, the assumption on the function $x \mapsto \beta(x) x$ is satisfied when $\beta$ is given by a Hill function (20). We can take

$$
\bar{x}=\frac{\theta}{(n-1)^{1 / n}} .
$$

Proposition 2. Assume that $\delta=0$ and (27) holds true. Let $v \geq \bar{x}$ be fixed, with $\bar{x}>0$ defined in Proposition 1. Consider the following initial condition

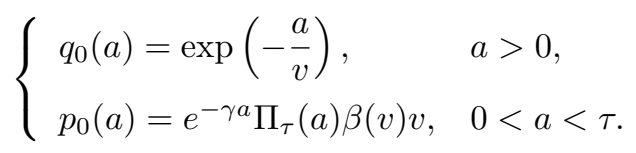

Then, $Q$ is an increasing function on $[0,+\infty)$ and $\lim _{t \rightarrow+\infty} Q(t)=+\infty$.

Proof. First, it is clear that $Q(0)=\int_{0}^{+\infty} \exp \left(-\frac{a}{v}\right) \mathrm{d} a=v>\bar{x}$. Second, the initial function $\phi$ is given by

$$
\phi(t)=\frac{e^{-\gamma t}}{\Pi_{\tau}(-t)} p_{0}(-t)=\beta(v) v, \quad t \in[-\tau, 0] .
$$


Then, from (7) we have

$$
\begin{aligned}
Q^{\prime}(0) & =-\beta(Q(0)) Q(0)+2(1-K) \int_{0}^{\tau} \frac{D_{\tau}(a)}{\Pi_{\tau}(a)} p_{0}(a) \mathrm{d} a \\
& =-\beta(Q(0)) Q(0)+2(1-K) \beta(v) v \int_{0}^{\tau} e^{-\gamma a} D_{\tau}(a) \mathrm{d} a \\
& =-\beta(Q(0)) Q(0)+2(1-K) \nu_{\gamma}(\tau) \beta(v) v .
\end{aligned}
$$

Consequently,

$$
\begin{aligned}
Q^{\prime}(0) & =\left(2(1-K) \nu_{\gamma}(\tau)-1\right) \beta(v) v \\
& >0
\end{aligned}
$$

So, $Q$ is increasing on $[0, \eta]$ for some $\eta \in(0, \tau]$. Suppose by contradiction that there exits $\eta \in(0, \tau]$ such that $Q^{\prime}(\eta)=0$ and $Q^{\prime}(t)>0$ for $t \in[0, \eta)$. Then, we have

$$
\begin{aligned}
0=Q^{\prime}(\eta) & \geq-\beta(Q(\eta)) Q(\eta)+2(1-K) \int_{\eta}^{\tau} e^{-\gamma \eta} \frac{D_{\tau}(a)}{\Pi_{\tau}(a-\eta)} p_{0}(a-\eta) \mathrm{d} a, \\
& \geq-\beta(Q(\eta)) Q(\eta)+2(1-K) \nu_{\gamma}(\tau) \beta(v) v .
\end{aligned}
$$

Moreover, $Q(\eta)>Q(0)=v \geq \bar{x}$. Then, $\beta(Q(\eta)) Q(\eta)<\beta(v) v$. Consequently,

$$
0=Q^{\prime}(\eta)>\left(2(1-K) \nu_{\gamma}(\tau)-1\right) \beta(v) v>0,
$$

which is absurd. We conclude that $Q$ is an increasing function on $[0, \tau]$. By direct application of the previous proposition, the result holds.

We note that the initial functions $q_{0}$ and $p_{0}$ in the previous proposition satisfy the assumption (4). But, they do not satisfy the compatibility condition (5). In fact, (4) is enough to provide a solution of (10)-(11).

\section{Discussion}

We proposed and analyzed an age-structured partial differential system describing the dynamics of hematopoietic stem cells (HSCs). We took into account the fact that a cell cycle has two phases, that is, HSCs are either in a quiescent phase or actively proliferating. We made the difference between dividing cells that enter directly to the quiescent phase (long-term proliferation) and dividing cells that return to the proliferating phase to divide again (short-term proliferation). These features were never been taken into account in previous models of HSCs dynamics. We focused our mathematical analysis on the behavior of the trivial steady state (global asymptotic stability) and the existence of unbounded solutions. We showed that the behavior of the system is related to both short and long proliferating terms. The model is relevant due to its ability to explain how an uncontrolled proliferation of HSCs can lead to either a high/low-throughput of HSCs. Thus, it can be able to characterize some hematological disorders, especially those associated to a high blood production (for instance, myeloproliferative

diseases) or a low blood production (for instance, anemias). A further analysis could help in understanding the complicated dynamics of the blood cell production system. 


\section{References}

[1] M. Adimy, A. Chekroun, and T.-M. Touaoula. A delay differential-difference system of hematopoietic stem cell dynamics. Comptes Rendus Mathematique, 353(4):303-307, 2015.

[2] M. Adimy and F. Crauste. Global stability of a partial differential equation with distributed delay due to cellular replication. Nonlinear Analysis: Theory, Methods $\&$ Applications, 54(8):1469-1491, 2003.

[3] M. Adimy and F. Crauste. Modelling and asymptotic stability of a growth factor-dependent stem cell dynamics model with distributed delay. Discrete and Continuous Dynamical Systems - Series B, 8(1):19-38, 2007.

[4] M. Adimy, F. Crauste, and A. El Abdllaoui. Discrete-maturity structured model of cell differentiation with applications to acute myelogenous leukemia. J. Biol. Syst., 16(03):395424, 2008.

[5] M. Adimy, F. Crauste, M. Hbid, and R. Qesmi. Stability and hopf bifurcation for a cell population model with state-dependent delay. SIAM J. Appl. Math., 70(5):1611-1633, 2010.

[6] M. Adimy, F. Crauste, and S. Ruan. A mathematical study of the hematopoiesis process with applications to chronic myelogenous leukemia. SIAM J. Appl. Math., 65(4):1328-1352, 2005.

[7] M. Adimy, F. Crauste, and S. Ruan. Stability and hopf bifurcation in a mathematical model of pluripotent stem cell dynamics. Nonlinear Analysis: Real World Applications, 6(4):651-670, 2005.

[8] M. Adimy, F. Crauste, and S. Ruan. Modelling hematopoiesis mediated by growth factors with applications to periodic hematological diseases. Bull. Math. Biol., 68(8):2321-2351, 2006 .

[9] W. Aiello, H. Freedman, and J. Wu. Analysis of a model representing stage-structured population growth with state-dependent time delay. SIAM J. Appl. Math., 52(3):855-869, 1992.

[10] G. B. Bradford, B. Williams, R. Rossi, and B. I. Quiescence, cycling, and turnover in the primitive hematopoietic stem cell compartment. Experimental hematology, 25(5):445-453, 1997.

[11] K. L. Cooke. Stability analysis for a vector disease model. Rocky Mountain J. Math., 9(1):31-42, 1979.

[12] M. A. Cruz and J. K. Hale. Stability of functional differential equations of neutral type. Journal of Differential Equations, 7(2):334-355, 1970.

[13] K. Gu and Y. Liu. Lyapunov-krasovskii functional for uniform stability of coupled differential-functional equations. Automatica, 45(3):798-804, 2009.

[14] M. E. Gurtin and R. C. MacCamy. Non-linear age-dependent population dynamics. Arch. Rational Mech. Anal., 54:281-300, 1974.

[15] J. K. Hale and M. A. Cruz. Existence, uniqueness and continuous dependence for hereditary systems. Annali di Matematica Pura ed Applicata, 85(1):63-81, 1970. 
[16] J. K. Hale and S. M. Verduyn Lunel. Introduction to Functional Differential Equations. Applied Mathematical Sciences 99, Springer-Verlag, New York, 1993.

[17] M. Iannelli. Mathematical theory of age-structured population dynamics, volume 7 of Applied mathematics monographs C.N.R. Giardini editori e stampatori, Pisa, 1995.

[18] Y. Kuang. Delay Differential Equations: With Applications in Population Dynamics. Mathematics in Science and Engineering, Academic Press, 1993.

[19] M. C. Mackey. Unified hypothesis for the origin of aplastic anemia and periodic hematopoiesis. Blood, 51(5):941-956, 1978.

[20] P. Pepe, Z.-P. Jiang, and E. Fridman. A new Lyapunov-Krasovskii methodology for coupled delay differential and difference equations. International Journal of Control, 81(1):107-115, 2008.

[21] L. Pujo-Menjouet, S. Bernard, and M. C. Mackey. Long period oscillations in a $\mathrm{G}_{0}$ model of hematopoietic stem cells. SIAM J. Appl. Dyn. Syst., 4(2):312-332, 2005.

[22] L. Pujo-Menjouet and M. C. Mackey. Contribution to the study of periodic chronic myelogenous leukemia. Comptes Rendus Biologies, 327(3):235-244, 2004.

[23] L. Sachs. The molecular control of hemopoiesis and leukemia. C.R. Acad. Sci. Paris. Serie III, 316(9):871-891, 1993.

[24] H. L. Smith. Reduction of structured population models to threshold-type delay equations and functional differential equations: a case study. Mathematical Biosciences, 113(1):1-23, 1993.

[25] H. Takizawa, R. R. Regoes, C. S. Boddupalli, S. Bonhoeffer, and M. G. Manz. Dynamic variation in cycling of hematopoietic stem cells in steady state and inflammation. $J$ Exp Med, 208(2):273-284, 2011.

[26] G. Webb. Theory of Nonlinear Age-Dependent Population Dynamics. Marcel Dekker Inc, New York and Basel, 1985. 